\title{
The Role of the Epinephrine Test in the Diagnosis and Management of Children Suspected of Having Congenital Long QT Syndrome
}

\author{
Sally-Ann B. Clur • Priya Chockalingam • Luc H. Filippini • \\ Ari P. Widyanti - Marc Van Cruijsen • Nico A. Blom • \\ Mariel Alders • Nynke Hofman • Arthur A. M. Wilde
}

Received: 4 September 2009/Accepted: 6 November 2009/Published online: 3 December 2009

(C) The Author(s) 2009. This article is published with open access at Springerlink.com

\begin{abstract}
The epinephrine test has been shown to be a powerful tool to predict the genotype of congenital long QT syndrome (LQTS). The aim of this study was to evaluate its role in the diagnosis and management of LQTS in children. The test (using the Shimizu protocol) was conducted in patients with some evidence of LQTS but in whom clinical and management decisions were challenging ( $n=41$, age $9.6 \pm 3.9$ years, 19 female). LQT1, LQT2, and negative responses to epinephrine were obtained in 16 , 5 , and 20 subjects, respectively. LQTS gene positivity was obtained in two subjects. Beta-blocker therapy was started in all subjects with a positive epinephrine response
\end{abstract}

S.-A. B. Clur $(\bowtie) \cdot$ P. Chockalingam · A. P. Widyanti ·

N. A. Blom

Department of Pediatric Cardiology, Emma Children's

Hospital, Academic Medical Centre, Meibergdreef 9,

Amsterdam 1105 AZ, The Netherlands

e-mail: s.a.clur@amc.uva.nl

S.-A. B. Clur · P. Chockalingam - N. A. Blom - A. A. M. Wilde The Centre for Congenital Heart Defects Amsterdam-Leiden,

Leiden, The Netherlands

P. Chockalingam - M. Van Cruijsen - A. A. M. Wilde

Heart Failure Research Centre, Department of Cardiology,

Academic Medical Centre, Amsterdam, The Netherlands

L. H. Filippini

Department of Pediatric Cardiology, Juliana Children's Hospital,

The Hague, The Netherlands

N. A. Blom

Department of Pediatric Cardiology, Leiden University Medical Centre, Leiden, The Netherlands

M. Alders · N. Hofman

Department of Clinical Genetics, Academic Medical Centre,

Amsterdam, The Netherlands $(n=21)$ and in some negative responders because of their strong LQTS phenotype $(n=10)$. No therapy was given to the subset with less convincing features of LQTS who had also responded negatively to epinephrine $(n=10)$. Follow-up for $3.0 \pm 2$ years was uneventful in both management groups. Due to the discordance with genotyping, the epinephrine test cannot be used to diagnose genotypepositive LQTS but when used in combination with phenotype assessment and genetic screening, it could enable better management decisions.

Keywords Long QT syndrome - Arrhythmia . Children · Diagnosis · Catecholamines · Genes · Beta-blocker therapy

\section{Introduction}

Congenital long QT syndrome (LQTS) is a primary inherited cardiac arrhythmia syndrome with an estimated prevalence of 1:2000 [2] and an annual mortality of $1 \%$ [11]. Various types of LQTS are associated with mutations in genes encoding distinct cardiac ion channels or membrane adaptors. The most common types, i.e., LQT1, LQT2, and LQT3, account for 85\% of genotyped cases [8]. LQTS may be dormant lifelong or may present with syncope, seizures, or sudden death from polymorphic ventricular tachycardia or torsades de pointes (TdP) and is one of the leading causes of sudden cardiac death (SCD) in children [21].

The diagnosis of LQTS has traditionally relied on the demonstration of a prolonged heart rate (HR) and a corrected QT interval (QTc) as well as the use of a clinical scoring system (Schwartz score, Keating criteria) that incorporates surface electrocardiographic (ECG) findings 
with clinical and family histories $[10,14,15]$. However, the sensitivity of the Schwartz and Keating criteria in identifying disease carriers has been shown to be low [8]. Although the typical LQTS cases present no diagnostic difficulty, the borderline cases are more complex, requiring the evaluation of multiple variables in addition to clinical history and surface ECG [1]. The fact that cardiac events are more often associated with sympathetic stimulation (physical or emotional stress) in LQT1 than in either LQT2 or LQT3 forms the basis of the epinephrine test. Studies by Shimizu et al. [16] and Vyas et al. [21, 22] have shown it to be a powerful tool to predict the genotype of LQT1, LQT2, and LQT3 syndromes. The present study was aimed at evaluating the role of the epinephrine test in an unbiased pediatric population with clinical suspicion of LQTS.

\section{Materials and Methods}

\section{Study Cohort}

The epinephrine test was conducted prospectively in 41 consecutive patients with clinical suspicion of LQTS between January 2003 and May 2008. The unifying factor in all of the subjects was a combination of clinical signs and symptoms that made both diagnosing as well as ruling out LQTS difficult. Hence, the epinephrine test was performed in addition to the routinely used tools to aid in the diagnostic process of LQTS. The male-to-female ratio was 22:19, and age ranged from 1 to 15 years at presentation.

\section{Study Design}

The assessment of subjects for LQTS involved a historic review, including family history, physical examination, resting ECG, 24-hour ECG, exercise test, epinephrine test, and LQTS genetic screening.

Emphasis was placed on the association of presenting symptoms with physical or psychological stress; on family history suggestive of LQTS, such as syncope, arrhythmia, and sudden unexplained death; and the relation of symptoms to the previously mentioned triggers.

Standard 12-lead ECG recorded with the subject at rest in the supine position was used to obtain baseline HR and QTc. The QT was manually measured by averaging three consecutive QRS complexes in precordial lead V5 (according to Shimizu et al. [16]). Bazett's formula $(\mathrm{QTc}=\mathrm{QT} / \sqrt{ } \mathrm{RR})$ was used to calculate the QTc. Resting QTc was considered prolonged if it was $\geq 460 \mathrm{~ms}$ ( $\geq 450 \mathrm{~ms}$ in male subjects) [15].

The Schwartz score, which is derived in part from the QTc, symptoms, and family history, was ascertained in all subjects. Definite LQTS is defined by an LQTS score $\geq 4 \quad(\leq 1$ point $=$ low probability of LQTS; $2-3$ points $=$ intermediate probability of LQTS; $\geq 4$ point$\mathrm{s}=$ high probability of LQTS) [15].

Criteria proposed by Keating were also used to assess the possibility of LQTS in the study cohort. According to these criteria, individuals are affected if they are asymptomatic with QTc $>470 \mathrm{~ms}$ or if they have typical symptoms with QTc $>450 \mathrm{~ms}$ [10].

The QTc from exercise ECG and/or 24-h ECG was used to gain additional diagnostic clues in some cases. Any prolongation in QTc above baseline value in the 24-h ECG was noted along with any rhythm disturbances [17]. Exercise test was performed using the Bruce protocol and a QTc $>450 \mathrm{~ms}$ at maximal exercise or at $1 \mathrm{~min}$ into recovery was taken as a positive result [9].

After the clinical assessment and the epinephrine challenge test, genetic screening for LQTS was performed in our laboratory for genetic analysis as outlined previously [8].

\section{Epinephrine Test}

The epinephrine test was performed using the protocol described by Shimizu et al. [16]. A bolus intravenous injection of epinephrine $(0.1 \mathrm{ug} / \mathrm{kg})$ was given under monitoring. This was immediately followed by a continuous infusion of epinephrine $(0.1 \mu \mathrm{g} / \mathrm{kg} / \mathrm{min})$ for $5 \mathrm{~min}$. A 12-lead ECG was recorded before the bolus, immediately after the bolus administration, and at 30-s intervals during the continuous infusion. Monitor surveillance was present throughout the test and for at least 15 min after stopping the infusion to monitor for the possible occurrence of TdP. Blood pressure was also monitored at 2-min intervals. The effect of epinephrine on the RR and QT intervals reaches steady-state at approximately $2-3 \mathrm{~min}$ after the start of the epinephrine infusion. Therefore, data representative of the peak epinephrine effect were obtained 1-2 min after the start of the infusion at peak HR, and data representative of the steady-state effect were collected at 3-5 min after the start of the infusion. According to Shimizu et al. [16], a subject was considered to have LQT1 response if the QTc increase in the peak phase was $\geq 35 \mathrm{~ms}$ and was maintained throughout the steady-state phase. LQT2 response was present if the peak QTc increase of $\geq 80 \mathrm{~ms}$ was not maintained in the steady-state phase. Both of these responses were considered positive. A lack of increase of QTc peak $\geq 35 \mathrm{~ms}$ was taken as a negative response. Subjects already taking beta-blockers at the time of the epinephrine test were instructed to stop the medication for at least 5 half-lives before the test to avoid confounding effects of the drug. 
Statistical Analysis

Continuous variables are presented as mean \pm SD and catagoric variables as number of patients $(n)$ and percentage $(\%)$. Student $t$ test was used to compare continuous data; $\chi^{2}$ Fisher's exact test was used for catagoric data; and $p \leq 0.05$ was considered statistically significant.

\section{Results}

Study Population

The study group consisted of 22 male and 19 female subjects of mean age $9.6 \pm 3.9$ years (range 1-15). The baseline HR was $80 \pm 15 \mathrm{bpm}$, and the baseline QTc was $441 \pm 28 \mathrm{~ms}$. Table 1 lists the phenotypic characteristics of the male and female subjects. None of the variables were statistically different between sexes.

\section{Historic Review}

The majority of subjects ( $n=30$ [73\%]) were symptomatic at presentation. Syncope (including near-drowning) and presyncope together formed the most common group, with 27 subjects $(66 \%)$ initially seeking medical attention for this reason. The other less commonly reported symptoms were palpitations ( $n=1$ [2\%]), "absence" seizurelike episodes $(n=1$ [2\%]), and out-of-hospital cardiac

Table 1 Phenotypic characteristics of the study population

\begin{tabular}{lll}
\hline Phenotypic characteristics & $\begin{array}{l}\text { Male } \\
(n=22)\end{array}$ & $\begin{array}{l}\text { Female } \\
(n=19)\end{array}$ \\
\hline $\begin{array}{l}\text { Age at presentation in years } \\
\text { (mean } \pm \text { SD) }\end{array}$ & $8.8 \pm 3.9$ & $10.5 \pm 3.5$ \\
$\begin{array}{l}\text { Age group in years (\%) } \\
5\end{array}$ & $6(27)$ & $1(5)$ \\
$6-10$ & $9(41)$ & $8(42)$ \\
$>10$ & $7(32)$ & $10(53)$ \\
No. symptomatic at presentation (\%) & $15(68)$ & $15(79)$ \\
No. incidental finding of prolonged & $4(18)$ & $3(16)$ \\
QTc $(\%)$ & $3(14)$ & $1(5)$ \\
No. family history leading to & & \\
suspicion of LQTS (\%) & $77 \pm 17$ & $84 \pm 13$ \\
Baseline HR in bpm (mean \pm SD) & & \\
Baseline QTc in ms (mean \pm SD) & $437 \pm 33$ & $447 \pm 23$ \\
Average Schwartz score (range) & $1.4(0-3)$ & $1.9(0-4)$ \\
No. Keating criteria positivity (\%) & $6(27)$ & $4(21)$
\end{tabular}

bpm beats per minute, $Q T c$ corrected QT interval

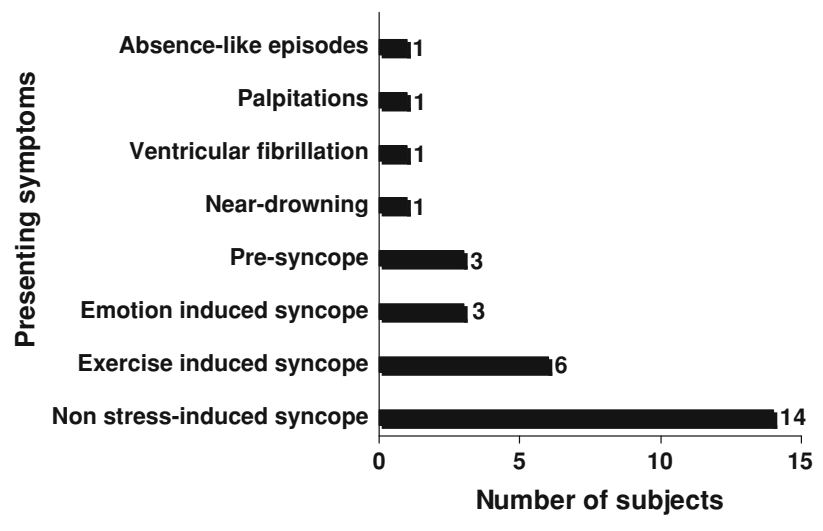

Fig. 1 Frequency of presenting symptoms in the study population

arrest due to ventricular fibrillation (VF) $(n=1[2 \%])$. Of the $11(27 \%)$ asymptomatic subjects, an incidental observation of prolonged QTc was made in 7 (17\%), whereas 4 (10\%) were thought to have LQTS based on suspicious family history. Figure 1 shows the frequency of the presenting symptoms in the study cohort. Family history was positive for SCD in two (5\%) families, arrhythmia (VF and/or documented TdP) in two (5\%) families, and unexplained syncope in two $(5 \%)$ families.

\section{QTc and Clinical Scoring}

The baseline QTc ranged from 380 to $502 \mathrm{~ms}$ (441 $\pm 28 \mathrm{~ms})$. Thirteen (32\%) subjects, 8 (61\%) of whom were symptomatic, had a significant QTc prolongation at baseline. Of the 26 subjects who had a 24-h ECG recording, $23(88 \%)$ had a documented QTc prolongation, especially with higher HRs. Exercise test was positive in 23 (68\%) of 34 subjects who underwent the test. No significant arrhythmias were noted during 24-h ECG monitoring and the exercise tests. In this study cohort, 18 (44\%) subjects had a Schwartz score $\leq 1 ; 22(54 \%)$ had a score of $2-$ 3 ; and $1(2 \%)$ had a score of 4 . The reason for suspicion of LQTS in subjects with a Schwartz score $\leq 1$ was unexplained syncope in eight subjects, exercise-related complaints of presyncope or palpitations in a setting of borderline QTc (440-460 ms) in five subjects, observation of borderline QTc in two subjects during follow-up for Kawasaki disease and pectus excavatum, respectively, and highly suspicious family history in three subjects. Keating criteria positivity was obtained in $10(24 \%)$ subjects.

Response to Epinephrine

Based on the changes in QTc with epinephrine administration, the subjects were divided into three groups. Sixteen (39\%) subjects had a QTc peak increase $\geq 35 \mathrm{~ms}$, which was maintained at steady-state (LQT1 response, group 1); 5 
Table 2 Comparison of clinical parameters of the three subject groups based on response to the epinephrine challenge test

bpm beats per minute, $Q T c$ corrected QT interval

\begin{tabular}{llll}
\hline Parameters & $\begin{array}{l}\text { LQT1 } \\
\text { response } \\
(n=16)\end{array}$ & $\begin{array}{l}\text { LQT2 } \\
\text { response } \\
(n=5)\end{array}$ & $\begin{array}{l}\text { Negative } \\
\text { response } \\
(n=20)\end{array}$ \\
\hline Baseline HR (bpm) & $74 \pm 10$ & $78 \pm 11$ & $87 \pm 18$ \\
Baseline QTc (ms) & $442 \pm 25$ & $435 \pm 40$ & $443 \pm 30$ \\
Increase in HR with epinephrine (bpm) & $29 \pm 9$ & $32 \pm 9$ & $20 \pm 10$ \\
Increase in QTc with epinephrine (ms) & $105 \pm 37$ & $100 \pm 11$ & $34 \pm 19$ \\
No. Schwartz score >3 (\%) & $0(0)$ & $1(20)$ & $0(0)$ \\
No. Schwartz score 2-3 (\%) & $9(56)$ & $2(40)$ & $11(55)$ \\
No. Schwartz score 1 (\%) & $7(44)$ & $2(40)$ & $9(45)$ \\
No. Keating criteria positivity (\%) & $2(13)$ & $1(20)$ & $7(35)$ \\
No. 24-h ECG positivity (\%) & $9(60)$ & $3(60)$ & $11(55)$ \\
No. exercise test positivity (\%) & $13(81)$ & $3(60)$ & $7(35)$ \\
No. genetic test positive (\%) & $1(6)$ & 0 & $1(5)$ \\
\hline
\end{tabular}

(12\%) subjects had a QTc peak increase $\geq 80 \mathrm{~ms}$, which was not maintained at steady-state (LQT2 response, group 2); and $20(49 \%)$ subjects did not show a QTc peak increase $\geq 35 \mathrm{~ms}$ (negative response, group 3 ). The average increase in QTc as well as in HR from baseline values was higher for the LQT1 and LQT2 response groups than for the negative response group (Table 2). Table 2 lists the clinical parameters of the LQT1 response, LQT2 response, and negative response groups.

\section{Complications During Epinephrine Test}

Two (5\%) subjects belonging to group 3 with baseline QTc of 450 and $479 \mathrm{~ms}$, respectively, experienced nonsustained ventricular tachycardia (NSVT) during the test.

\section{Genetic Screening}

Based on clinical suspicion, genetic screening for LQTS was performed in 39 of the 41 subjects. A 14-year-old female subject with history of an episode of syncope (normal ECG, QTc $439 \mathrm{~ms}$ ) demonstrated classic hyperventilation during the epinephrine test and had a negative response to the test. She was diagnosed with hyperventilation syndrome. A 9-year-old male subject who was screened for LQTS due to the sudden death of his father was not found to have any clinical indicators of LQTS (asymptomatic, normal ECG, QTc $428 \mathrm{~ms}$, and negative epinephrine response). Hence, the previously mentioned two subjects did not undergo genetic analysis for LQTS.

The LQTS genes tested were KCNQ1 (LQT1, $n=39$ ), $\mathrm{KCNH} 2$ (LQT2, $n=39$ ), SCN5A (LQT3, $n=25$ ), KCNE1 (LQT5, $n=20$ ), KCNE2 (LQT6, $n=19$ ), and KCNJ2 (LQT7, $n=1$ ) (Fig. 2). Only when appropriate (based on clinical history), subjects underwent screening for RYR2 and CASQ2 (genes associated with catecholaminergic

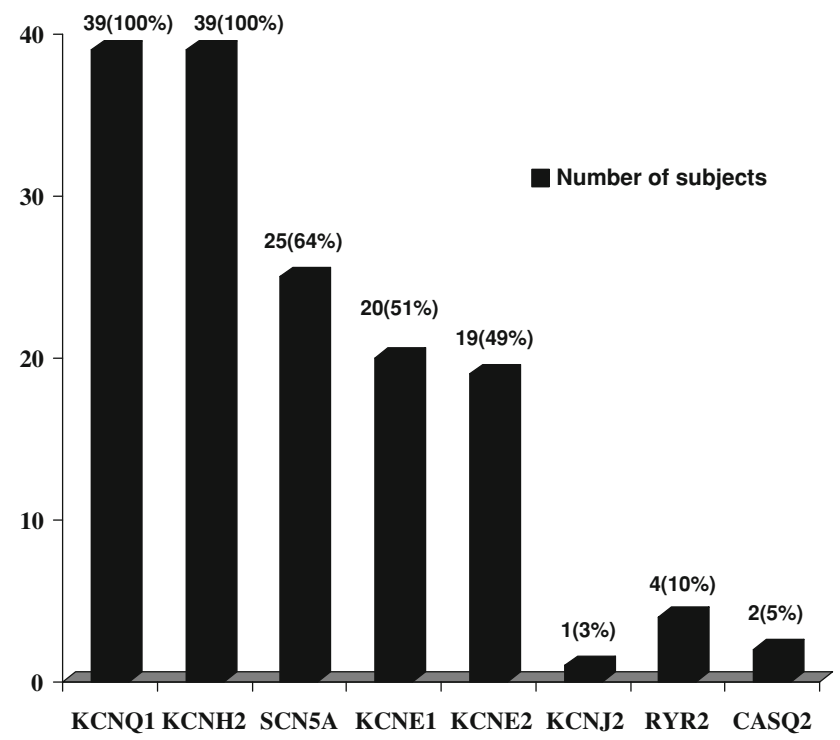

Fig. 2 Number of subjects screened for the different LQTS and CPVT genes: KCNQ1-LQT1 gene, KCNH2-LQT2 gene, SCN5ALQT3 gene, KCNE1-LQT5 gene, KCNE2-LQT6 gene, KCNJ2-LQT7 gene, RYR2 and CASQ2-CPVT genes

polymorphic ventricular tachycardia or CPVT). Pathogenic KCNQ1 mutations were shown in two subjects. A KCNQ1gene mutation was detected (p.Phe193Leu) in a 15-year-old female subject with recurrent syncope, baseline QTc $440 \mathrm{~ms}$, Schwartz score 1, and LQT1 response to epinephrine. A 9-year-old female subject with syncope, baseline QTc $470 \mathrm{~ms}$, Schwartz score 3, negative epinephrine response, and initially negative genetic screening underwent further extensive DNA analysis using multiplex ligationdependent probe amplification (MLPA), which detected a large KCNQ1 deletion mutation of exons 2-10.

Furthermore, two mutations of questionable pathogenicity were found in a 3-year-old male subject with syncope, baseline QTc $427 \mathrm{~ms}$, Schwartz score 1, and negative 
epinephrine response. The first is a variant c.3331-9-8 delGT in intron 14 of the $\mathrm{KCNH} 2$ gene, and the other is mutation c.895T $>\mathrm{A}$ in the SCN5A gene.

A 10-year-old female subject with a near-drowning episode and a previous history of Wilms' tumour was noted to have a slightly abnormal methylation of the L1T1 gene, a gene associated with Beckwith-Wiedemann syndrome. She had a LQT1 type epinephrine response, and her LQTS screening (LQT1, 2, 3, 5 and 6) was negative.

An 8-year-old female subject with Turner syndrome, prolonged QTc on routine cardiac evaluation, and LQT1type epinephrine response was also negative for LQTS screening (LQT1, 2, 3, 5, and 6).

\section{Management and Follow-Up}

Beta-blocker therapy was instituted to all subjects with a positive epinephrine response (group1 + group 2, $n=21$ ) and to $50 \%$ of the negative responders $(n=10)$ based on persisting suspicion of LQTS (gene positivity in one symptomatic subject, two mutations of unknown pathogenicity in one symptomatic subject, significantly abnormal ECG with prolonged QTc in five subjects, and ventricular ectopic activity and/or NSVT in three subjects). The rationale for no therapy in $50 \%$ of the negative responders ( $n=10$ ) was the "virtual" ruling out of LQTS by a negative epinephrine response, particularly in combination with a less convincing phenotype. Beta-blockers were either withdrawn after the epinephrine test response (four subjects), or the initial decision for no therapy was maintained after the test ( $n=6$, including the subject with VF who was implanted with an implantable cardiac defibrillator (ICD) because his genetic screening for LQTS and CPVT was negative).

Mean baseline QTc and Schwartz scores of the therapy group (446 \pm 29 and $1.7 \pm 1.1$, respectively) were higher than those of the nontherapy group (426 \pm 28 and $1.4 \pm 1.0$, respectively). All of the patients undergo regular pediatric cardiology clinic follow-up (maximum 8 years; mean $3.0 \pm 2$ years; age $12.8 \pm 3.7$ years) with periodic 24-h ECG monitoring and exercise tests. The subjects in the therapy group were compliant and responded well to treatment. They had no problems related to beta-blockers side effects. The subjects in the nontherapy group were free of LQTS-related cardiac events.

\section{Discussion}

The LQTS phenotype is highly variable, ranging from asymptomatic ECG repolarisation abnormalities to sudden death. Male sex, a history of syncope at any time during childhood, and a QTc duration $>500 \mathrm{~ms}$ have been shown to be the three main risk factors for life-threatening cardiac events in children with LQTS who are $<12$ years of age [6]. Early identification of patients at risk and a reliable diagnosis of LQTS become extremely important for effective management of these children. Although diagnostic tools-such as clinical scoring, provocation tests, and molecular screening-have all contributed to identifying LQTS in patients, a "gold-standard" diagnostic tool is still lacking for this challenging group of disorders. Although molecular diagnosis of LQTS, which is not always similar to confirmation, has now become commercially available, only approximately $70 \%$ of families with a suspected channelopathy are noted, whereas the remaining families with a strong clinical probability of LQTS will have a negative genetic test result [18-20]. Furthermore, it has been shown that a negative genetic test in a subject with clinical LQTS (i.e., genotype-negative/ phenotype-positive LQTS) does not provide a basis to discard the diagnosis [5].

In asymptomatic LQTS patients with normal repolarisation values (silent mutation carriers), the clinical scoring loses efficacy. Provocation tests, such as the epinephrine test, are proposed to have added value as an additional diagnostic and predictive tool in this setting. Landmark studies on the value of epinephrine testing in the diagnosis of LQTS have been published by Shimizu et al. [16] and Vyas et al. [21, 22]. During the epinephrine test, QTc prolongation at the peak of epinephrine, which is maintained at steady state conditions of epinephrine, has been described in patients with LQT1. Epinephrine prolongs the QTc more dramatically at the peak of epinephrine infusion in LQT2 patients, but the QTc returns to baseline levels at steady-state conditions. A much milder prolongation of QTc at the peak of epinephrine has been described in LQT3 patients and controls, and it returns to the baseline levels at steady-state conditions. Based on the pathophysiology of these genotypes, this response is reasonably well understood [16]. Thus the epinephrine test in patients suspected with LQTS was proposed to assist in the identification of affected patients, to allow for the prediction of the genetic type, and to guide the order of molecular DNA analysis. Hence, we used the test as a tool to guide clinical decision making in a pediatric cohort with a suspicious LQTS phenotype.

Recently, Magnano et al. showed that catecholamines, such as epinephrine and isoproterenol, are associated with significant QTc prolongation also in healthy subjects [13], which in turn questions the diagnostic efficacy of the epinephrine test. The results of the present study show epinephrine test sensitivity and specificity of 50 and $61 \%$, respectively, for the LQT1 response subset. The positive predictive value for subsequent identification of a positive genotype in the major potassium channel genes (in 
particular LQT1) is $6 \%$, and the negative predictive value for (virtually) ruling it out is $96 \%$. The lower test values. compared with the study by Vyas et al. [22]. may be explained by the difference in the referral pattern between the two institutions as well as in the age group of the subjects.

Based on recent advances in LQTS genotyping, it was hypothesized that approximately $70 \%$ of subjects with a positive epinephrine response would show LQTS gene positivity. Contrary to expectations, only $5 \%$ (1 of 21 ) of positive responders did have an underlying LQTS mutation. The paradox between the epinephrine test and genotyping could possibly be attributed to the following factors: The present study included all children with a suspicious LQTS phenotype and did not have specific inclusion criteria based on QTc and clinical scoring compared with the more rigid inclusion criteria adopted by Shimizu et al. [16] (LQTS-affected individuals were noted on the basis of electrocardiographic diagnostic criteria by Keating et al., including a QTc $\geq 470 \mathrm{~ms}$ in asymptomatic individuals and a QTc $>440 \mathrm{~ms}$ for men and $>460 \mathrm{~ms}$ for women associated with one or more of the following: stress-related syncope, documented TdP, or family history of early SDC). This paradox may also reflect differences in our population or minor differences in the infusion protocols. Moreover, on pursuing genetic testing with the MLPA technique, we identified a deletion mutation in an LQT1 patient with an impressive LQTS phenotype. There could be other such cases eluding genotype at the present time, thus accounting for the lower-than-expected positive yield.

However, the response to epinephrine has enabled the management of this challenging group of patients. It should once again be highlighted here that the study cohort, which was comprised of young patients with a constellation of signs and symptoms suggestive but not conclusive of LQTS, made diagnostic and management decisions difficult. The subjects who responded positively to epinephrine were considered likely to benefit from beta-blockers and hence were started on therapy. A diagnosis of LQTS was "virtually" ruled out in 50\% of negative responders based on their response to epinephrine combined with their less convincing clinical phenotype; hence, they were not commenced on beta-blockers.

The therapy and nontherapy groups were both followedup at regular intervals. Although the subjects using betablockers responded well to treatment, the subjects in the no-therapy group did not show any LQTS-related cardiac events warranting inclusion of therapy. Being able to rule out LQTS with confidence is important because a positive diagnosis brings with it enormous emotional, social, and economic consequences. Uncertainty of the diagnosis leads to anxiety for both families and caregivers [7]. This study shows that the epinephrine test together with clinical scoring and genetic screening helps in making a more confident management decision.

An abnormal exercise test has been shown to have a high correlation with LQT1 subtype [9]. In the current study, LQT1 responders had a high concordance of $81 \%$ with exercise test positivity; LQT2 responders had a $60 \%$ concordance; and negative responders had a low concordance of $35 \%$ (Table 2). Although it could be argued that both the exercise and epinephrine tests appear to produce similar results and could be performed mutually exclusively, it is also evident that both tests have some value in this difficult situation of positively diagnosing suspicious LQTS. The exercise test is noninvasive, relatively easier to perform, and may be useful to expose a prolonged QTc in borderline cases, whereas the epinephrine test might prove a better tool to rule LQTS in suspicious cases.

The numerous mutations in the LQTS-associated genes reported to date are point mutations or small insertions and deletions in coding regions or at splice junctions. It has recently been shown that large, multiple-exon deletions and duplications in cardiac ion channel genes account for a noteworthy proportion of LQTS cases [3, 12]. In the present study, MLPA DNA analysis eventually identified a large pathogenic KCNQ1 gene deletion in an 8-year-old female subject.

The therapeutic options of this genotypically and phenotypically heterogeneous disease include beta-blockade, mexiletine, pacemakers, ablation of the left stellate ganglion, ICD implantation, in addition to sports restrictions and avoidance of medications that lengthen repolarisation [4]. ICD therapy is reserved for high-risk patients unlikely to respond to who have failed betablocker therapy. All of the subjects in this study who were in the therapy group responded adequately to betablockers. Device implantation was not warranted in any of the subjects except in the infant diagnosed with idiopathic ventricular fibrillation.

The epinephrine test is generally a safe test with a low rate of complications. Ventricular extrasystoles, ventricular bigeminy, NSVT, and spontaneously terminating TdP have been reported. The present study shows the test to be safe in children with NSVT, which was noted in only $2(5 \%)$ subjects.

\section{Study Limitations}

The study is limited by the lack of a control group. It would be ideal to compare the responses to epinephrine in (more definite) pediatric LQTS patients with those in healthy children, but the ethical issues involved in such a study are paramount. 


\section{Conclusion}

Congenital LQTS in children is a potentially life-threatening disease. Although effective therapeutic strategies for LQTS have evolved, it is still challenging at times to make the appropriate diagnostic and management decisions in suspected cases. It is clear that the epinephrine test cannot be used to diagnose genotype-positive LQT, but when used in combination with phenotype assessment and genetic screening, it could enable better management decisions.

Open Access This article is distributed under the terms of the Creative Commons Attribution Noncommercial License which permits any noncommercial use, distribution, and reproduction in any medium, provided the original author(s) and source are credited.

\section{References}

1. Crotti L, Celano G, Dagradi F, Schwartz PJ (2008) Congenital long QT syndrome. Orphanet J Rare Dis 3:18

2. Crotti L, Stramba-Badiale M, Pedrazzini M, Ferrandi C, Insolia R, Matteo PS et al (2005) Prevalence of the long QT syndrome. Circulation 112:U724

3. Eddy CA, MacCormick JM, Chung SK, Crawford JR, Love DR, Rees MI et al (2008) Identification of large gene deletions and duplications in KCNQ1 and KCNH2 in patients with long QT syndrome. Heart Rhythm 5:1275-1281

4. Etheridge SP, Sanatani S, Cohen MI, Albaro CA, Saarel EV, Bradley DJ (2007) Long QT syndrome in children in the era of implantable defibrillators. J Am Coll Cardiol 50:1335-1340

5. Goldenberg I, Moss AJ (2008) Long QT syndrome. J Am Coll Cardiol 51:2291-2300

6. Goldenberg I, Moss AJ, Peterson DR, McNitt S, Zareba W, Andrews ML et al (2008) Risk factors for aborted cardiac arrest and sudden cardiac death in children with the congenital long-QT syndrome. Circulation 117:2184-2191

7. Hendriks KS, Grosfeld FJ, Wilde AA, van den Bout J, van Langen IM, van Tintelen JP et al (2005) High distress in parents whose children undergo predictive testing for long QT syndrome. Community Genet 8:103-113

8. Hofman N, Wilde AA, Tan HL (2007) Diagnostic criteria for congenital long QT syndrome in the era of molecular genetics: do we need a scoring system? Eur Heart J 28:1399
9. Kaufman ES, Gorodeski EZ, Dettmer MM, Dikshteyn M (2005) Use of autonomic maneuvers to probe phenotype/genotype discordance in congenital long QT syndrome. Am J Cardiol 96:1425-1430

10. Keating M, Atkinson D, Dunn C, Timothy K, Vincent GM, Leppert M (1991) Linkage of a cardiac arrhythmia, the long QT syndrome, and the Harvey ras-1 gene. Science 252:704-706

11. Keating MT, Sanguinetti MC (2001) Molecular and cellular mechanisms of cardiac arrhythmias. Cell 104:569-580

12. Koopmann TT, Alders M, Jongbloed RJ, Guerrero S, Mannens MM, Wilde AA et al (2006) Long QT syndrome caused by a large duplication in the KCNH2 (HERG) gene undetectable by current polymerase chain reaction-based exon-scanning methodologies. Heart Rhythm 3:52-55

13. Magnano AR, Talathoti N, Hallur R, Bloomfield DM, Garan H (2006) Sympathomimetic infusion and cardiac repolarization: the normative effects of epinephrine and isoproterenol in healthy subjects. J Cardiovasc Electrophysiol 17:983-989

14. Schwartz PJ (1985) Idiopathic long QT syndrome: progress and questions. Am Heart J 109:399-411

15. Schwartz PJ, Moss AJ, Vincent GM, Crampton RS (1993) Diagnostic criteria for the long QT syndrome. An update. Circulation 88:782-784

16. Shimizu W, Noda T, Takaki H, Kurita T, Nagaya N, Satomi K et al (2003) Epinephrine unmasks latent mutation carriers with LQT1 form of congenital long-QT syndrome. J Am Coll Cardiol 41:633-642

17. Sugao M, Fujiki A, Sakabe M, Nishida K, Tsuneda T, Iwamoto J et al (2006) New quantitative methods for evaluation of dynamic changes in QT interval on 24-hour Holter ECG recordings: QT interval in idiopathic ventricular fibrillation and long QT syndrome. Heart 92:201-207

18. Tester DJ, Ackerman MJ (2005) Genetic testing for cardiac channelopathies: ten questions regarding clinical considerations for heart rhythm allied professionals. Heart Rhythm 2:675-677

19. Tester DJ, Will ML, Haglund CM, Ackerman MJ (2006) Effect of clinical phenotype on yield of long QT syndrome genetic testing. J Am Coll Cardiol 47:764-768

20. van Langen IM, Birnie E, Alders M, Jongbloed RJ, Le Marec H, Wilde AA (2003) The use of genotype-phenotype correlations in mutation analysis for the long QT syndrome. J Med Genet 40:141-145

21. Vyas H, Ackerman MJ (2006) Epinephrine QT stress testing in congenital long QT syndrome. J Electrocardiol 39:S107-S113

22. Vyas H, Hejlik J, Ackerman MJ (2006) Epinephrine QT stress testing in the evaluation of congenital long-QT syndrome: diagnostic accuracy of the paradoxical QT response. Circulation 113:1385-1392 